\title{
Midwakh associated Acute Lung Injury (MALI): An emerging epidemic in the Middle East -A Case Report and review of literature
}

\author{
Angel Varughese ${ }^{1}$, Suneel Pooboni ${ }^{2}$, Vijay Vinod ${ }^{1}$, and Rania Abusamra ${ }^{3}$ \\ ${ }^{1}$ Mediclinic City Hospital \\ ${ }^{2}$ Mediclinic Middle East \\ ${ }^{3}$ Mohammed Bin Rashid University of Medicine and Health Sciences Hamdan Bin \\ Mohammed College of Dental Medicine
}

September 5, 2020

\begin{abstract}
Tobacco in any form is harmful. There is an alarming increase consumption of Alternative Tobacco Products (ATPs). Midwakh is one of the most used ATP in UAE, particularly amongst adolescents. In addition to harmful levels of cobalt, chromium, cadmium, Iron and lead, Midwakh has higher nicotine and tar content when compared to shisha and cigarettes. We report a 14-year-old adolescent male, who presented with Acute Lung Injury within 24 hours following a first attempt of Midwakh smoking, a presentation remarkably similar to E-Cigarettes or Vaping Associated Lung Injury. A high-resolution computerized tomogram of the chest (HRCT) showed bilateral interstitial pneumonia with ground glass appearance and patchy consolidation. A flexible bronchoscopy revealed bilateral petechial hemorrhages with erythematous bronchial walls. Mechanical ventilation was required for two weeks with high ventilatory settings due to poor lung compliance. The criteria for moderately severe ARDS was met, and a good response was achieved to a high dose of steroids, prone ventilation with ultra-short lung protective strategy. Initial spirometry tests done post extubation were compatible with severe restrictive lung disease. Significant clinical and radiological recovery was achieved at three months. To our knowledge and as per the literature reviewed, this is the first case of Midwakh associated acute lung injury reported till date. We wish to emphasize that physicians should be well informed about the use of ATPs, both existing and emerging ones and its potential severe complications.
\end{abstract}

\section{INTRODUCTION}

Tobacco is one of the leading causes of death in the world ${ }^{3}$. The prevalence of adolescent smoking is alarming, with around 24 million children (13-15 years) worldwide, of which 13 million of them use smokeless tobacco products ${ }^{3}$ Exposure of nicotine during adolescence or childhood has deleterious effects on brain development, as smoking leads to impaired lung function and growth. As per the WHO Global Youth and Adult Tobacco Survey, there is an alarming increase and a growing concern of Alternative Tobacco Products $(\text { ATPs })^{3,4,5}$.Common ATPs range from Electronic Cigarettes, Hookah or Shisha, Midwakh or Dokha, and various Smokeless Tobacco products. Midwakh and Dokha are two terms that are often used interchangeably.

Acute Lung Injury (ALI) and Acute Respiratory Distress Syndrome (ARDS) cause significant morbidity and mortality among the affected, particularly the younger generation. Smoking exposes the lungs to severe detrimental effects on epithelial and endothelial function, quite similar to ALI.

In 2020, over 2800 E-Cigarettes or Vaping Associated Lung Injury (EVALI)cases of hospitalization or deaths have been reported to the Centre for Disease Control (CDC|) from all 50 states in the USA, with ages ranging from $13-85$ years 6 . 
We report a 14-year-old adolescent male, who presented with signs and symptoms of ALI / ARDS following a single session of Midwakh smoking, a presentation very similar to EVALI. To our knowledge, this is the first case of Midwakh Associated Acute Lung Injury (MALI) ever reported to date.

\section{CASE PRESENTATION}

A 14-year-old boy, previously fit and healthy, presented to his local hospital in March 2020 with rapidly developing respiratory symptoms of breathlessness, cough, and fever over 24 hours post smoking Midwakh, mixed with crushed Damas tree leaves (Conocarpus Lancifolius). On admission, he underwent a series of investigations including COVID 19; started on empirical broad-spectrum antibiotics and oseltamivir, and underwent a high-resolution computerized tomogram of the chest (HRCT), which demonstrated interstitial pneumonia with patchy consolidation and ground-glass appearance (figure 1). Within a few hours, he required intubation and mechanical ventilation due to significant hypoxia.

On day four of mechanical ventilation, he was transferred to our center on Volume control-Assist control settings (FiO2 $40-70 \%$, PEEP 8, Tidal volume $400 \mathrm{ml}$ ). Initial arterial blood gas upon admission revealed relative hypoxemia ( $\mathrm{PaO} 277.9, \mathrm{PCO} 242.3 \mathrm{~mm} \mathrm{Hg})$. The patient met the criteria for moderately severe ARDS (Bilateral infiltrates on radiographs, $\mathrm{PaO} 2 / \mathrm{FiO} 2$ ratio $192 \mathrm{~mm} \mathrm{Hg}(<200)$ on PEEP 8 and no cardiogenic cause). He was managed with ultra-short lung-protective ventilation ( $\mathrm{Tv} \sim 5 \mathrm{ml} / \mathrm{kg}$ ), given poor lung compliance. Neuromuscular blockage and sedation were administered. Auscultation of the chest revealed bilateral decreased air entry and crackles. Continuous infusion of low dose furosemide was commenced for a positive cumulative fluid balance of 4 liters. During his admission, regular chest physiotherapy was performed.

Flexible bronchoscopy was performed on day 5, which revealed severe bilateral inflammation, petechial hemorrhages, and erythematous bronchial walls beside mild increase of airway secretions, in keeping with fulminant lung injury. Bronchoalveolar lavage had negative microbiology culture, viral PCR including the COVID-19 PCR and cell count differentiation of $71 \%$ neutrophils, $5 \%$ Lymphocytes, $6 \%$ monocytes, $18 \%$ macrophages, and no eosinophils. A diagnosis of interstitial pneumonitis with ARDS due to inhaled toxins was considered, for which the patient was commenced on a five day course of the following anti-inflammatory medications; intravenous Methyl Prednisolone one gram/day (15 mg/kg/day), oral hydroxychloroquine (300 mg per day) and oral azithromycin (500 mg per day). During the PICU admission course, he developed hypertension and pre-renal failure for which anti-hypertensive medications were administered, and diuretics were discontinued. The patient was rehydrated following this episode, balancing the perfusion of his kidneys and lungs. Antibiotics were rationalized because of negative microbiology results. On day 10, high-pressure ventilation was still required despite the high dosage of methylprednisolone. A repeat chest HRCT scan (figure 2) was performed, which revealed left lower lobe consolidation and right lower lobe collapse and the interstitial lung changes with ground glass appearances. Subsequently, a prolonged period of prone ventilation was initiated, after which there was a significant improvement of the ventilator requirements until extubation on day 14. Spirometry was done five days post-extubation and revealed a restrictive ventilatory pattern with forced vital capacity (FVC), Forced expiratory volume in one second (FEV1), and FEV1/FVC being $2.15 \mathrm{~L}$ $(46.8 \%), 2.02 \mathrm{~L}(53.3 \%)$ and $94.09 \%(112.8 \%)$ respectively. A minimal global weakness, features of critical illness myopathy and peripheral neuropathy were noted. The patient was discharged on a tapering dose of Prednisone.

Follow up at three months post-discharge showed significant recovery with resolving respiratory symptoms and improving effort tolerance. A follow-up HRCT Chest (figure 3) at three months post-discharge was normal, Spirometry has significantly improved with FVC of $3.8 \mathrm{~L}$ (91\% predicted), FEV1 of $3.1 \mathrm{~L}$ (89\% predicted), slightly reduced Diffusion capacity (DLCO) of $6.3 \mathrm{~L}$ (70\% predicted) and normal 6 min walk test was obtained (with a heart rate of $110 \mathrm{bpm}$ before a walk and $121 \mathrm{bpm}$ after. Oxygen saturation (SpO2) of $100 \%$ before the walk and $98 \%$ after).

\section{DISCUSSION}

We present a patient with ARDS due to acute lung injury related to Midwakh. Dokha is manufactured 
by drying tobacco leaves, mixed with herbs and spices to enhance the flavor. It is smoked through a small pipe called Midwakh, which has three parts, a mouthpiece, a stem, and a bowl (image 1). The bowl can contain approximately 0.5 grams of dry tobacco, enough to finish one session in 2 inhalations; Therefore, an individual ends up smoking Midwakh 20-25 times a day.

Smoking Midwakh is increasing in the Gulf and particularly in the $\mathrm{UAE}^{7}$, among the adolescent and school students ${ }^{1}$.It is the most commonly used ATP in UAE, next only to Cigarette on overall tobacco use ${ }^{8}$. Nicotine levels in Midwakh tobacco products were $170.76 \%, 218.07 \%, 128.57 \%$, and $193.33 \%$ higher in comparison with cigarettes, chewing tobacco, snuff tobacco, and electronic cigarettes respectively and its tar content is the highest when compared to shisha and cigarettes ${ }^{2}$. Midwakh tobacco products contains harmful levels of cobalt, chromium, cadmium, iron, and lead. In addition to these, carcinogens and Central nervous system (CNS) depressants were found ${ }^{9,10}$.Traditional Midwakh pipes contain no filters; hence these toxicants can quickly enter the lungs. Midwakh smokers may add herbs, spices, dried flowers, fruits, bark, and leaves of native plants (like the Damas tree leaves) to tobacco. Nevertheless, the effects on these combinations and the byproducts on combustion have never been reported.

Toxic inhalational lung injury may result in a spectrum of respiratory disorders like bronchopneumonia, pulmonary edema, interstitial lung disease, reactive airways dysfunction syndrome, obstructive airway disease, and Bronchiolitis Obliterans Organizing Pneumonia (BOOP), as the triggering agents could be single or multiple. Fatal cases following significant exposure were associated with laryngeal edema, airway obstruction, non-cardiogenic pulmonary edema/ARDS, and secondary infection in the setting of the diffuse airway and pulmonary parenchymal damage ${ }^{11}$. They also described the resolution of initial acute pneumonia to be followed by the gradual onset of airway obstruction, which has to be watched during the follow-up.

The clinical presentation and radiological findings, including CT scans, were quite similar to the Ecigarette/Vaping-associated Lung Injury (EVALI) outbreak in the USA. Patients with EVALI present with acute, severe respiratory distress with findings typical of a chemical-induced pneumonitis after using ECigarette or vaping products ${ }^{12}$. Symptoms like cough, shortness of breath, fever can rapidly worsen within 24-48 hours of initial presentation, as happened in our case $\mathrm{e}^{13}$.

In addition to antibiotics, patients with EVALI were treated by systemic steroids to reduce the inflammatory response. As per the CDC, steroids showed an improvement in the clinical condition in almost $82 \%$ of patients treated ${ }^{14}$. CDC recommends follow up at 1-2 weeks (SpO2 and chest x-ray). Some patients have relapsed during corticosteroid tapering, and some have had persistent hypoxemia ( $\mathrm{SpO} 2<95 \%)$ requiring home oxygen at discharge ${ }^{14}$.

ARDS has an incidence of $30-80$ cases per 100,000 populations ${ }^{15}$. It is divided into three categories as per Berlin definition from 2012 according to severity of hypoxemia as mild ( $\mathrm{PaO} 2 / \mathrm{FiO} 2$ 200-300 mmHg), moderate $(\mathrm{PaO} 2 / \mathrm{FiO} 2$ 100-200 $\mathrm{mmHg})$ and severe $(\mathrm{PaO} 2 / \mathrm{FiO} 2<100 \mathrm{mmHg})$ forms of ARDS.

Despite a much-improved understanding of ARDS pathophysiology, the efficacy of used therapeutic approaches is limited. In our case, poor compliance of the lungs despite the low requirement of FiO2 up to $50 \%$ was remarkable. For a set tidal volume of $5 \mathrm{ml} / \mathrm{kg}$, his peak inspiratory pressure was reaching up and beyond $35 \mathrm{~cm} \mathrm{H} 2 \mathrm{O}$. Thus we resort to ultra-protective lung ventilation with tidal volumes of 4 to $5 \mathrm{ml} / \mathrm{kg}$ as required to avoid barotrauma. Prone positioning was useful especially in the acute phase of severe ARDS, by enabling better ventilation/perfusion matching, more homogenous distribution of ventilation with reduction of Ventilator-induced lung injury and recruitment of dorsal regions ${ }^{16}$.

The use of neuromuscular blocking agents (NMBA) improves patient-ventilator synchrony and reduces oxygen consumption leading to improved survival ${ }^{17}$. However, when given alone with steroids, it can exacerbate weakness due to critical illness myopathy and polyneuropathy ${ }^{18}$.In our case, it could not be avoided as we had to balance the risk of ICU-acquired weakness with the potential benefit of neuromuscular blockers and systemic glucocorticoids in the severe lung injured patient.

Lee et al. highlighted various new outcomes that need to be studied in Pediatric Acute Respiratory Distress 
Syndrome (PARDS) like long term pulmonary function, risk of pulmonary hypertension, nutrition status and growth, PICU acquired weakness, neurocognitive development, functional status and Health-related quality of life (HRQOL) ${ }^{19}$. A cohort study conducted on 316 mechanically ventilated pediatric patients concluded that $23 \%$ developed new morbidities due to residual organ dysfunction, treatment complications etc ${ }^{20}$.

There have been numerous studies on long term outcomes in adult ARDS. One study with potential application to pediatrics illustrated that half of the patients had persistent functional disability 12 months after discharge $^{21}$. The 6 -minute walk test (6MWT) helps assess global physical function, i.e., lung and cardiac function and muscle strength. The mean distance walked in the $6 \mathrm{MWT}$ increased significantly over the first year when followed up at three months and 9-12 months after discharge ${ }^{22}$.

Our patient had significantly reduced spirometry parameters upon discharge from the PICU, which got almost normalized within three months post-discharge from the hospital.

\section{CONCLUSION}

We report the first-ever case of Midwakh associated Acute Lung Injury (MALI), which posed a diagnostic challenge during the COVID-19 pandemic period. It was a diagnosis of exclusion and was based on a history of clinical presentation, signs and symptoms, radiological features. It was a close mimic of the recent EVALI epidemic in the USA. Our case responded to a high dose of Steroids, Prone ventilation with ultra-short lung-protective settings. Significant recovery was achieved at three months though the long-term effects have to be watched for.

As of 2020, there are no such clinical studies on Midwakh to identify long term risks attributable to it. We recommend additional research and studies to identify the pathophysiology of acute lung injury, acute and long-term effects of Midwakh smoking. We request physicians and pediatricians to work closely with adolescents and their parents to discourage tobacco products. Appropriate public health education and strictly obeying the country's tobacco control laws can prevent a MALI epidemic in the region and protect the younger generation.

\section{REFERENCES}

1. Crookes A, Wolff K. Prevalence of the tobacco product dokha among high school students in Dubai. Subst Use Misuse. 2014 Aug; 49(10):1349-52.

2. Mahboub, Bassam, Mohammad, Ayesha Begum et al. Analytical Determination of Nicotine and Tar Levels in Various Dokha and Shisha Tobacco Products. Journal of Analytical Toxicology. 2018;42 (7): 496-502.

3. World Health Organization (WHO). Report on the global tobacco epidemic: warning about the dangers of tobacco. Geneva: WHO, 2011.

4. Centers for Disease Control and Prevention (CDC). Current tobacco use among middle and high school students-United States, 2011. MMWR Morb Mortal Wkly Rep 2012; 61:581-5.

5. Morton J, Song Y, Fouad H, et al. Cross-country comparison of water pipe use: nationally representative data from 13 low and middle-income countries from The Global Adult Tobacco Survey (GATS). Tob Control 2014; 23:419-27.

6. Outbreak of Lung Injury Associated with the Use of E-Cigarette, or Vaping, Products. (2020, February 25). Retrieved July 12, 2020, from https://www.cdc.gov/tobacco/basic_information/ecigarettes/severe-lung-disease.html

7. Jayakumary M, Jayadevan S, Ranade AV, Mathew E .Prevalence and pattern of dokha use among medical and allied health students in Ajman, United Arab Emirates. Asian Pac J Cancer Prev ,2010 ;11: 1547-1549.

8. Al-Houqani M, Ali R, Hajat Tobacco smoking using Medwakh is an emerging health problem - evidence from a large cross-sectional survey in the United Arab Emirates. PLoS ONE 2012; 7:e39189.

9. Elsayed, Yehya; Dalibalta, Sarah; El Kouche, Maissam .Chemical characterization and safety assessment of dokha: An emerging alternative tobacco product. Science of the Total Environment. 2018 Feb 15;615:9-14. 
10. Bassam Mahboub, Mohmmad El Hajjaj et al. Analytical Determination of Heavy Metals in Various Dokha and Shisha Products, and Related Health Implications. Biomedical Journal of Scientific \& Technical Research. 2019;23(5):17829-17835

11. Deji Adegunsoye; Samaan Rafeq: Toxic Inhalational Lung Injury: Pulmonary medicine: Pulmonology advisor June 29, 2019

12. David C. Christiani , M.D., M.P.H. Vaping-Induced Acute Lung Injury The New England Journal of Medicine March 5, 2020; 382:960-962

13. Harold J. Farber New Understanding of the Health Hazards of Electronic cigarettes and Vaping. Pediarics in Review Vol.41,Issue 3.1 Mar 2020

14. Siegel DA, Jatlaoui TC, Koumans EH et al Update: Interim Guidance for Health Care Providers Evaluating and Caring for Patients with Suspected E-cigarette, or Vaping, Product Use Associated Lung Injury — United States,October 2019 Morbidity and Mortality weekly report (MMWR).2019; 68(41);919-927

15. Standiford T.J., Ward P.A. Therapeutic targeting of acute lung injury and acute respiratory distress syndrome. Transl. Res. 2016;167:183-191.

16. Gattinoni L., Taccone P., Carlesso E., Marini J.J. Prone position in acute respiratory distress syndrome. Rationale, indications, and limits. Am. J. Respir. Crit. Care Med. 2013;188:1286-1293.

17. Neto A.S., Pereira V.G., Espósito D.C., Damasceno M.C., Schultz M.J. Neuromuscular blocking agents in patients with acute respiratory distress syndrome: A summary of the current evidence from three randomized controlled trials. Ann. Intensive Care. 2012; Jul 26;2(1):33.

18. Hanson P., Dive A., Brucher J.M., Bisteau M., Dangoisse M., Deltombe T. Acute corticosteroid myopathy in intensive care patients. Muscle Nerve. 1997; 20(11):1371-80.

19. Lee S.W.,Loh S.W.,Ong C.,Lee J.H.Pertinent clinical outcomes in pediatric survivors of pediatric acute respiratory distress syndrome (PARDS): a narrative review. Ann Transl Med.2019 Oct;7(19):513.

20. Keim G, Watson RS, Thomas NJ, et al. New Morbidity and Discharge Disposition of Pediatric Acute Respiratory Distress Syndrome Survivors. Crit Care Med 2018;46:1731-8.

21. Herridge MS, Tansey CM, Matte' A, Tomlinson G, Diaz-Granados N, Cooper A, et al. Functional disability 5 years after acute respiratory distress syndrome. N Engl J Med 2011 Apr 7;364(14):1293304.

22. Chakdour S, Vaidya PC, Angurana SK, et al. Pulmonary Functions in Children Ventilated for Acute Hypoxemic Respiratory Failure. Pediatr Crit Care Med 2018; Sep;19(9):e464-e471. 

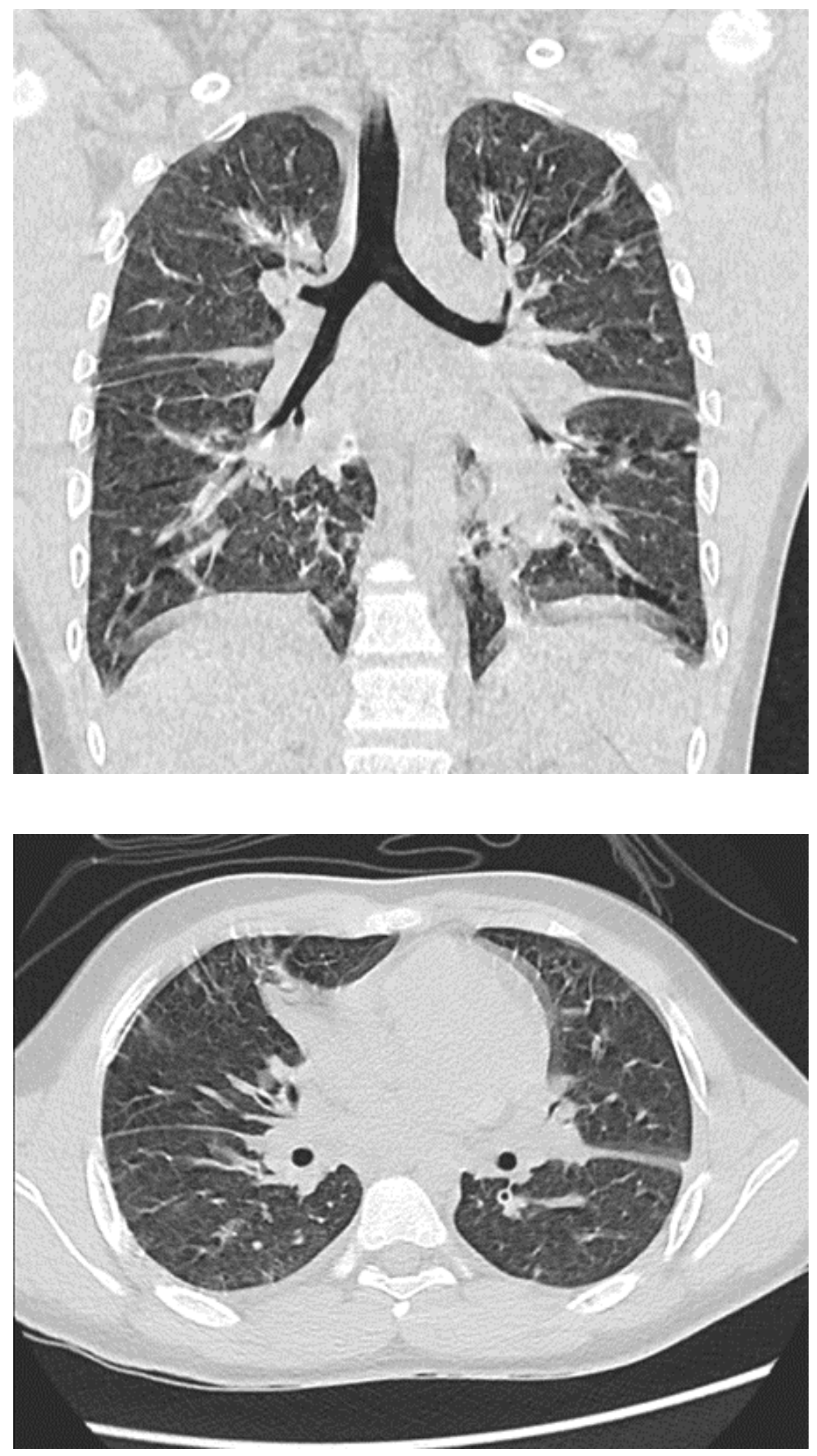


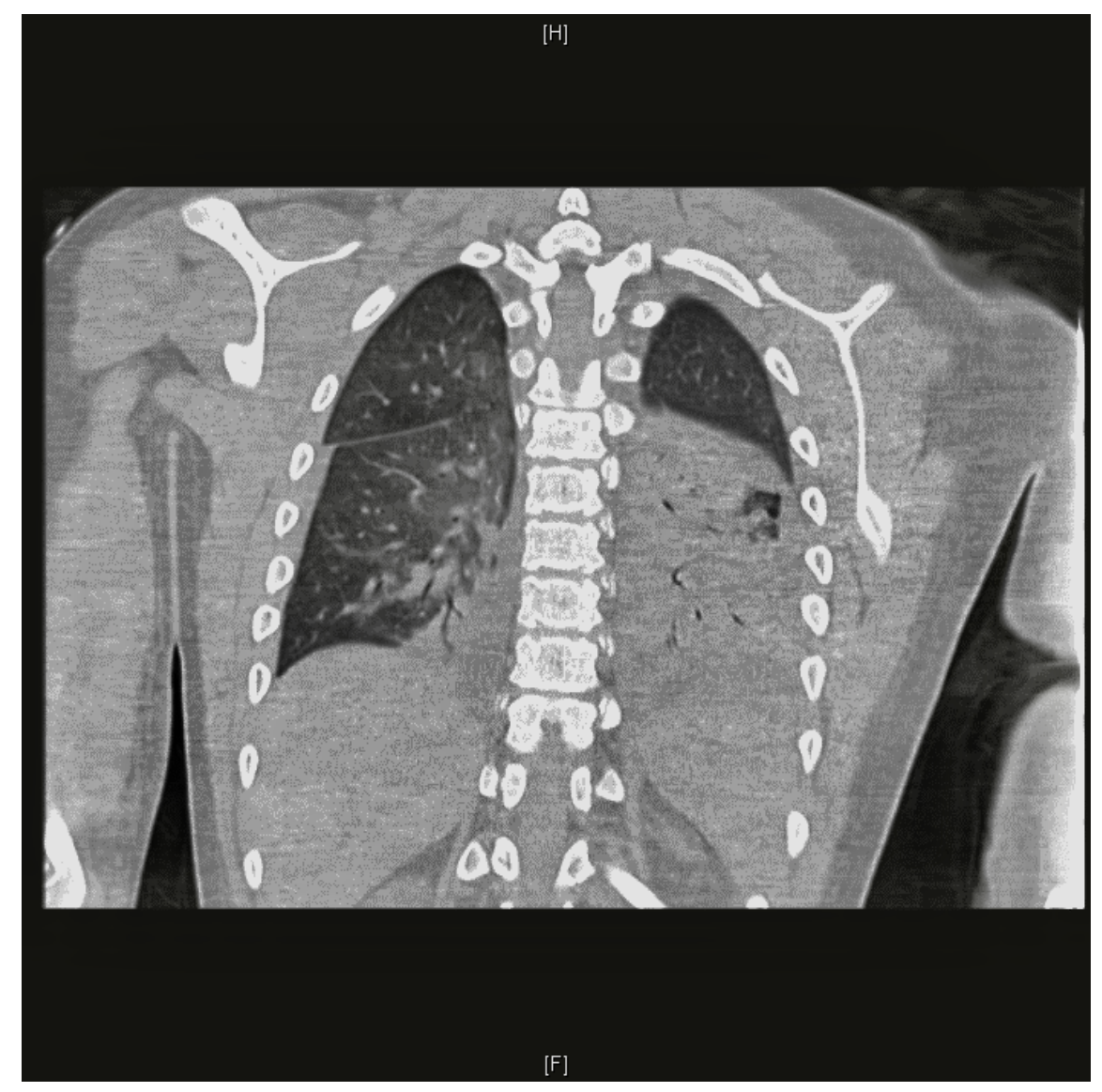




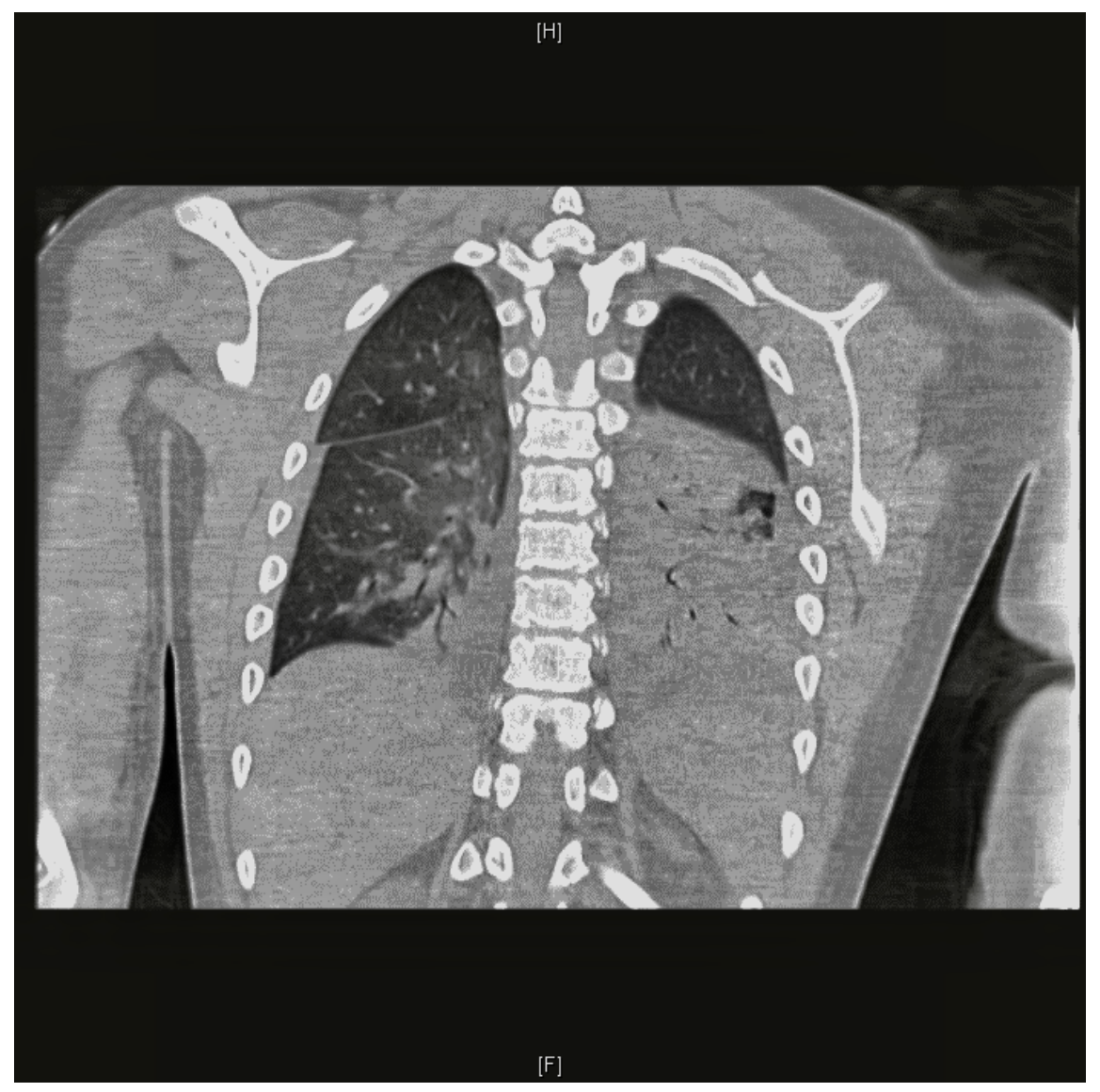




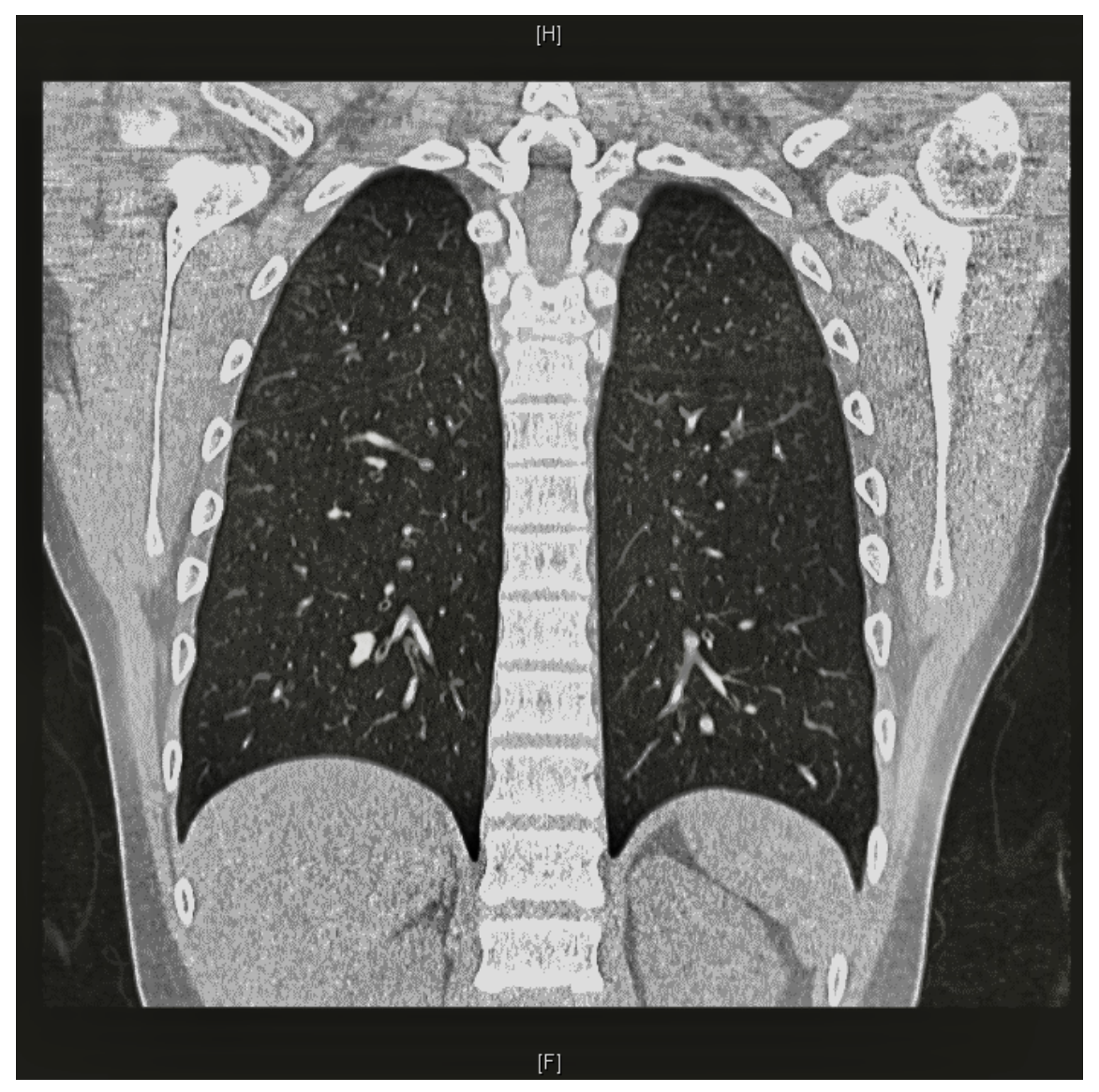



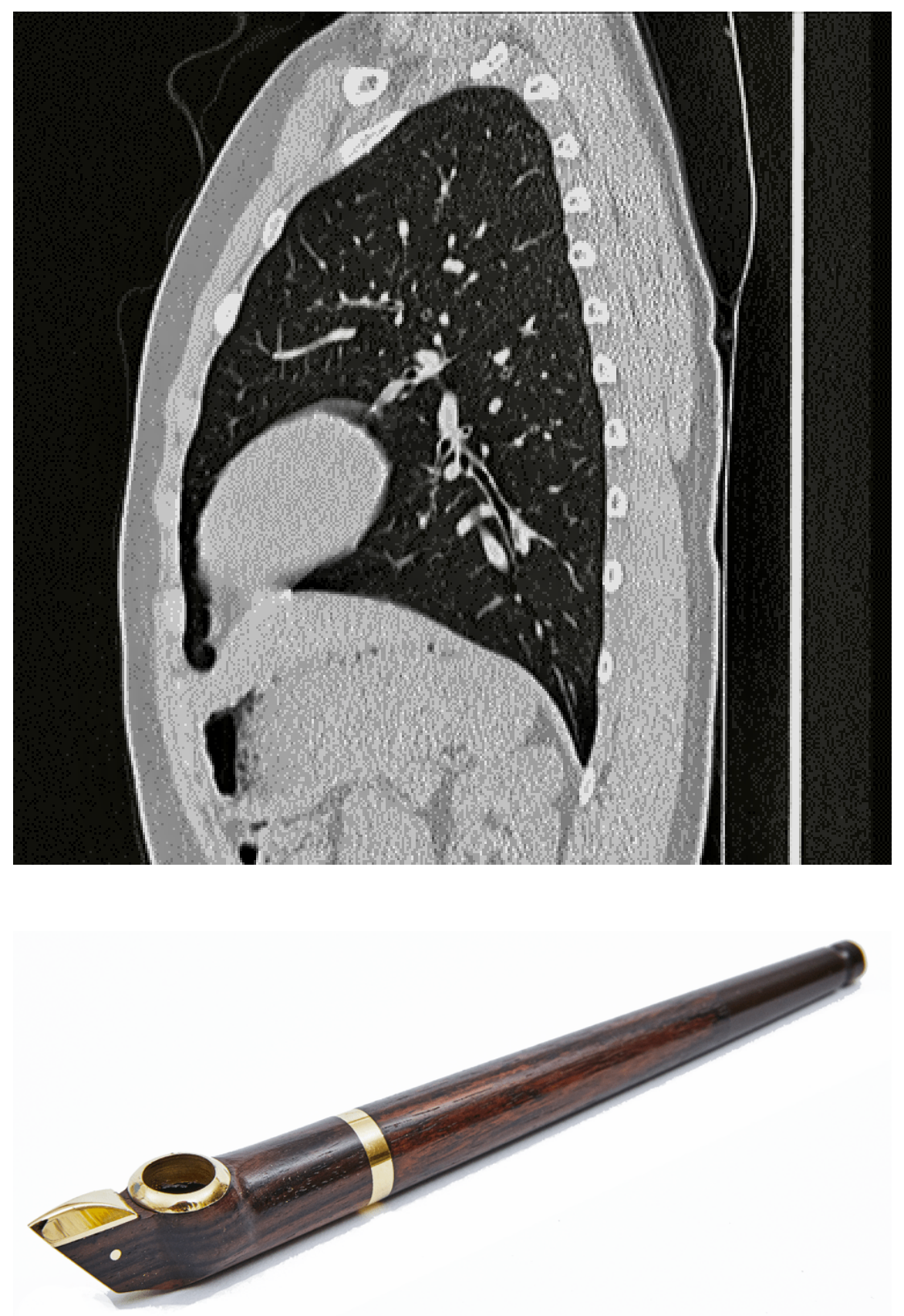


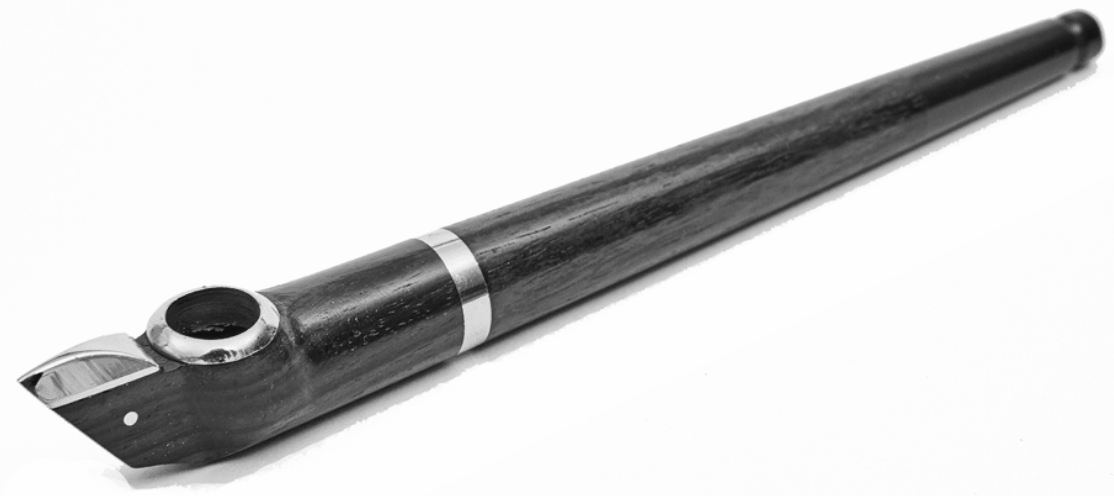

